(C) <2019>. This manuscript version is made available under the CC-BY-NC-ND 4.0 license http://creativecommons.org/licenses/by-nc-nd/4.0/

The definitive publisher version is available online at 10.1016/j.techfore.2018.12.025 


\title{
Forecasting technical emergence: An introduction
}

\author{
Yi Zhang ${ }^{1}$, Alan Porter $^{2,3}$, Denise Chiavetta ${ }^{3}$, Nils C. Newman ${ }^{3}$, Ying Guo ${ }^{4}$ \\ ${ }^{1}$ Centre for Artificial Intelligence, Faculty of Engineering and Information Technology, University of \\ Technology Sydney, Sydney, Australia; \\ ${ }^{2}$ Technology Policy and Assessment Center, Georgia Institute of Technology, Atlanta, USA \\ ${ }^{3}$ Search Technology Inc., Norcross, USA \\ ${ }^{4}$ School of Management and Economics, Beijing Institute of Technology, Beijing, China \\ Email: yi.zhang@uts.edu.au; alan.porter@isye.gatech.edu; dchiavetta@searchtech.com; \\ newman@searchtech.com; guoying_bit@163.com.
}

The FUSE program, Foresight and Understanding from Scientific Exposition, of the Intelligence Advanced Research Projects Activity (IARPA) sparked interest in measuring technical emergence. This special issue focuses on research contributing to development of measures (indicators) of "tech emergence." It draws on papers presented at special tech emergence sessions at the 2017 Portland International Conference on Management of Engineering and Technology (PICMET) in Portland, Oregon, USA, and at the 2017 Global Tech Mining (GTM) Conference in Atlanta, Georgia, USA. These sixteen papers offer a trove of conceptual and empirical research aimed at tracking, assessing, and projecting emergence of specific technologies. ${ }^{1}$

We approach "technical emergence" from a broad perspective of science, technology, \& innovation (ST\&I) - e.g., advances in scientific development and in technical evolution, as well as in emergent commercial innovations. Understanding processes of technical emergence becomes essential for technological forecasting investigations at either macro or micro levels - e.g., technology roadmapping, technology delivery system modelling, approaches to identify drivers of technical emergence, and other perspectives such as empirical assessment to validate prior forecasts. Specifically, interests in measuring technical emergence align with this special issue in three discernible categories:

- Identifying reproducible and useful indicators of technical emergence are a prime interest moving forward. Machine learning, network analysis, or other approaches that advance the ability of such data analytics offer potential for emergence indicators. Secondary indicators that effectively connect those topical indicators with players (e.g., countries, regions, organizations, and individuals) to uncover who are actively engaging emerging technical topics are also vital. Treatment of complementary data and measures offers promise - e.g.,

\footnotetext{
${ }^{1}$ Our efforts in editing this volume and several of the papers have benefited from support of the U.S. National Science Foundation (NSF). We acknowledge NSF support (Award \#1759960 - "Indicators of Technological Emergence") to Search Technology and Georgia Tech. The findings and observations contained in this paper and the special issue are those of the authors and do not necessarily reflect the views of the National Science Foundation.
} 
ways to tap $R \& D$ community growth and funding patterns, or possibly stakeholder opposition to emerging sciences and technologies.

- Profiling technical emergence entails representation of varied dimensions attendant to rapidly growing elements within an $\mathrm{R} \& \mathrm{D}$ domain. Ways to identify topical content and to track and project its evolution hold strong appeal.

- Efforts to forecast emergence are of keen concern. How does one go beyond time series analyses and tracking advances? Either quantitative or qualitative solutions, especially mixed methods, would be advantageous.

We briefly introduce the sixteen submissions collected in this special issue and spotlight points of special interest using these three categories.

Indicators and secondary indicators of technical emergence emphasize the identification of emergent terms and topics, and their association with multiple entities, such as countries, regions, organizations, and individuals. Empirical insights hidden behind the interactions among such indicators and specific emerging sectors are also explored in a series of case studies.

Porter et al. propose a method in the paper entitled Emergence scoring to identify frontier $R \& D$ topics and key players to calculate emergence scores for topical terms derived from scientific documents, and a family of such indicators are applied for identifying cutting-edge countries, organizations, and individuals in a target $\mathrm{R} \& \mathrm{D}$ domain.

By considering technological convergence as the key to understand technical emergence, Kose and Sakata develop a module-based mining method to recognize patterns of technological convergence in the paper Identifying technology convergence in the field of robotics research. Wang et al. integrate technological convergence with the analytic framework of emerging scores for extracting emergent topics in their paper, entitled An approach to identify emergent topics of technological convergence: A case study for $3 D$ printing.

Jeong, Park and Yoon extend the focus of technical emergence from emerging topics to emerging research and business development areas, and their paper, entitled Identifying emerging research and business development $(R \& B D)$ areas based on topic modeling and visualization with intellectual property right, proposes an integrated framework with topic modeling and network analysis for uncovering such R\&BD areas and potential technological opportunities. In parallel, Ma et al. concentrate on the clinical translation of scientific discoveries from bench to bedside in the paper entitled Identifying translational indicators and technology opportunities for nanomedical research using tech mining: The case of gold nanostructures, and a series of indicators are designed to profile research activities and forecast potential trends.

The involvement of other concepts and instruments in the area of technology management in investigating technical emergence is also recognized. Based on the concept of technological speciation, Moehrle and Caferoglu construct a method to detect emerging technologies from mainstream technologies by using a series of variables for semantically characterizing emerging technologies in the paper, Technological speciation as source for emerging technologies. Using semantic patent analysis for the case of camera technology. Berg et al. build on the understanding of technological innovation systems to introduce two patent 
classification code-based indicators for investigating patent trajectories and categorical concentration, and then help identify first signals of emerging dominance in the paper entitled Identifying first signals of emerging dominance in a technological innovation system: A novel approach based on patents.

Profiling technical emergence includes discovering the content of technical emergence and tracking the dynamics of technical emergence. Such efforts have gained complementary benefits with the involvement of technology roadmapping and its variations, such as tracking emerging trends and innovation/evolutionary pathways.

Suominen, Peng, and Ranaei focus on the dynamics of research networks (e.g., research participation and community involvement) during the development of emerging technologies through a case study in the paper Examining the dynamics of an emerging research network using the case of triboelectric nanogenerators. Li et al. integrate patents and social media data (e.g., Twitter) to investigate the dynamics of emerging technologies - e.g., how technologies emerge and develop in Identifying and monitoring the development trends of emerging technologies using patent analysis and Twitter data mining: the case of perovskite solar cell technology.

Forecasting innovation pathways (FIP) has been recognized as an effective tool for profiling historical data and foreseeing technical advances. Robinson, Lagnau, and Boon present an addition to the FIP toolbox that helps characterise and demarcate boundaries of emerging fields to allow for deeper analysis in Innovation pathways in additive manufacturing: Methods for tracing emerging and branching paths from rapid prototyping to alternative applications. Huang et al. integrate a series of text mining techniques (e.g., term clumping, subject-actionobject analysis, and network analysis) within the framework of FIP, aimed at identifying key $\mathrm{R} \& \mathrm{D}$ areas and tracing technological evolution over the time in the paper, Tracing the system transformations and innovation pathways of an emerging technology: Solid lipid nanoparticles.

The engagement of streaming data analytics with science maps creates a new angle for mapping technological evolution. In the paper Discovering interactions in big data research: A learningenhanced bibliometric study, Zhang et al. apply a method called scientific evolutionary pathways for profiling technological landscapes and identifying the evolutionary relationships among technological topics in the area of big data techniques.

Forecasting emergence becomes crucial for understanding technical emergence and applying such intelligence to take pre-emptive actions in national $R \& D$ and enterprise strategic management. Advanced information technologies, such as machine learning and complex network analysis, are effectively involved in such endeavors.

As a bibliometric mainstream for forecasting technical emergence, citation patterns and their structural changes are exploited as the key for identifying new scientific breakthroughs in the paper Searching for new breakthroughs in science: How effective are computerised detection algorithms written by Winnink, Tijssen, and van Raan.

Analyzing the topological structures of bibliometric networks (e.g., citation/co-citation, coauthorship, and co-word networks) has been recognized as an effective way to grasp the dynamics of such networks. In the paper Early identification of important patents: Design and 
validation of citation network metrics, Mariani, Medo, and Lafond propose an age-normalized measure of patent centrality to identify significant patents through citation network analysis, and thus to predict potential emerging technologies.

Qualitative approach-based forecasting studies are involved as well. In the paper A method for anticipating the disruptive nature of digitalization in the machine-building industry, Sommarberg and Makinen exploit a visual analogue scale to utilize expert knowledge in foreseeing potential disruptions in industry sectors.

Validating prior forecasting studies provides value-added insights for data analysts, ST\&I researchers, and stakeholders in related emerging sectors. In the paper An assessment of technology forecasting: Revisiting earlier analyses on dye-sensitized solar cells (DSSCs), Huang et al. revisit the results of a 10-year dye sensitized solar cell-based forecasting study conducted by the Innovation Co-Lab (Science, Technology and Innovation Program, Georgia Institute of Technology, Manchester Institute of Innovation Research, University of Manchester, and the School of Management and Economics, Beijing Institute of Technology).

In reflecting on the contributions, this special issue highlights endeavours on measuring and forecasting technical emergence, contributed by the communities of bibliometrics and technology management. We can specify such efforts in three categories - i.e., indicators and secondary indicators of technical emergence, profiling technical emergence, and forecasting technical emergence. Despite the promise of qualitative approaches, this special issue indicates stronger interest in the use of new empirical techniques, concepts, and their applications. That is, text mining techniques, in particular topic models, have been widely exploited to understand scientific documents in profiling technical emergence. Network analyses complement traditional bibliometrics to contribute to forecasting technical emergence. Machine learning techniques have become an effective tool for enhancing the capability of bibliometric approaches in handling complicated issues of technological forecasting. How best to integrate such advanced information technologies for investigating technical emergence could be a direction for future studies. Again, we welcome suggestions for any further development of forecasting technical emergence down the line. 